\title{
Comparative Study of Conductivity in Mid-Voltage Cable XLPE Insulation
}

\author{
F. Frutos ${ }^{1}$, M. Acedo ${ }^{1}$, A. Jadraque ${ }^{1}$, M. Mudarra ${ }^{2 *}$, J. Sellarès ${ }^{2}$ and J. Belana ${ }^{2}$ \\ ${ }^{1}$ Universidad de Sevilla, Depto. Física Aplicada I, ETS Ingeniería Informática, \\ Avda. Reina Mercedes s/n, Sevilla 41012, Spain \\ ${ }^{2}$ Universitat Politècnica de Catalunya, Campus de Terrassa, Dept. Física i Enginyeria Nuclear, \\ C. Colom, 11, Terrassa, 08222, Spain \\ ${ }^{*}$ E-mail : miguel.mudarra@upc.es
}

\begin{abstract}
It is well established that space charge conditions insulation lifetime. Because of this, space charge formation and relaxation processes characterization has become a very active research field, not only from an basic science point of view, but also in order to improve insulation performance in cable and other devices.

In the case of mid-voltage power cable, crosslinked polyethylene is widely used as insulator. Service temperature of such cables is around $90^{\circ} \mathrm{C}$, which in the case of the cable insulation studied is in the melting temperature range. In previous works it has been determined that at this temperature conductivity plays a relevant role in the space charge relaxation process, so that the characterization of conductive processes at service temperature may become important in order to enhance cable insulation performance. The aim of this work is to contribute to a better knowledge of the conductive properties of XLPE in both time and frequency domains.
\end{abstract}

\section{INTRODUCTION}

Cable for power distribution has a limited lifetime. A central issue for cable manfacturers and power distribution companies is to predict when is the most convenient time to replace an aged cable on service, before it fails, in order to increase their customer satisfaction. Cable insulation is one of the elements that conditions cable lifetime mostly. In the case of mid-voltage power cable crosslinked polyethylene (XLPE) is widely used as insulation due to its suitable mechanical and electrical characteristics.

Most of the tecniques that have been proposed for cable insulation diagnosis rely on its electrical properties. Therefore, so that a better understanding of charge transport, trapping and relaxation processes in high insulating materials is essential to improve the aplicability of those techniques. The aim of this paper is to contribute to a better knowledge of the conductive properties of XLPE cable insulation by means of absortion/resorption currents and dynamic electric analysis measurements.

Time-domain measurements: absortion/resorption currents
The current density in a dielectric can be expressed by the equation

$$
\begin{aligned}
& J(t)=\sigma E(t)+\varepsilon_{0} \varepsilon_{r} \frac{d E}{d t}+ \\
& \varepsilon_{0} \int_{-\infty}^{\infty} f(\tau) \frac{d E(t-\tau)}{d t} d \tau
\end{aligned}
$$

where $f(\tau)$ is the dielectric response function associated with polarization processes. In the case that the dielectric material is subjected to a rectangular pulse shaped voltage from the time $t$ to $t+T$, the applied electric field can be expressed as $E(t)=E_{0}[u(t)-u(t-T)]$, where $u(t)$ is the step function. Thus, the resulting current density is given by:

$$
\begin{array}{r}
J=\sigma E(t)+\varepsilon_{0} \varepsilon_{r} E_{0}[\delta(t)-\delta(t-T)]+ \\
\varepsilon_{0} E_{0}[f(t)-f(t-T)]
\end{array}
$$

The absortion current, $J_{a}$ is the current along the time interval $[t, T)$,

$$
J_{a}=\sigma E_{0}+\varepsilon_{0} \varepsilon_{r} E_{0} \delta(t)+\varepsilon_{0} E_{0} f(t)
$$

and the resorption current, $J$ is the current along the interval $[T, \infty)$,

$$
J_{r}=-\varepsilon_{0} \varepsilon_{r} E_{0} \delta(t-T)+\varepsilon_{0} E_{0}[f(t)-f(t-T)]
$$

Using the capacity of the sample, $C_{0}$ and the applied voltage across it, $U$, the conductivity can be expressed after a long enough time by the equation:

$$
\sigma \approx \frac{\varepsilon_{0}}{C_{0} U}\left(I_{a}(t)+I_{r}(t)\right)
$$

where $I_{a}$ and $I_{r}$ refer to the absortion and resorption currents respectively.

Frequency-domain measurements: Sublinear dispersive conductivity

In some materials it has been observed a sublinear dispersive conductivity of the form:

$$
\sigma(\omega)=\sigma_{0}+A \omega^{n}
$$

where $\sigma_{0}$ is the dc conductivity, $A$ is a temperature dependent parameter and $n$ is a fractional exponent which 
ranges between 0 and 1 and has been interpreted by means of many body interactions among charge carriers. This behavior, termed universal dynamic response, has been observed in highly disordered materials like ionically conducting glasses, polymers, amorphous semiconductors and also in doped crystalline solids[1]. Eq. (6) can be derived from the "universal" dielectric response function for the dielectric loss of materials with free hopping carriers, and this derivation allows one to understand the temperature dependence of the $A$ parameter[2].

Power-law dependencies of conductivity, as in the case of Eq. (6), imply a power-law dependence of the form $(\mathrm{j} \omega)^{n}$ for the complex conductivity[1]. Therefore, this magnitude can be written as:

$$
\sigma^{*}(\omega)=\sigma_{0}+A(\mathrm{j} \omega)^{n}+\mathrm{j} \omega \varepsilon_{0} \varepsilon_{\infty}
$$

A crossover frequency $\omega_{p}$ can be defined as $\omega_{p}^{n}=\sigma_{0} / A$, so that Eq. (7) can be rewritten as

$$
\sigma^{*}(\omega)=\sigma_{0}+\sigma_{0}\left(\mathrm{j} \frac{\omega}{\omega_{p}}\right)^{n}+\mathrm{j} \omega \varepsilon_{0} \varepsilon_{\infty}
$$

This frequency $\omega_{p}$ is associated with the crossover from the power-law dependence observed at high frequency to a frequency independent dc regime that occurs at low frequencies. The real and imaginary parts of the conductivity are respectively:

$$
\begin{gathered}
\sigma^{\prime}(\omega)=\sigma_{0}+\sigma_{0} \cos \left(n \frac{\pi}{2}\right)\left(\frac{\omega}{\omega_{p}}\right)^{n} \\
\sigma^{\prime \prime}(\omega)=\sigma_{0} \sin \left(n \frac{\pi}{2}\right)\left(\frac{\omega}{\omega_{p}}\right)^{n}+\omega \varepsilon_{0} \varepsilon_{\infty}
\end{gathered}
$$

\section{EXPERIMENTAL}

In the case of time domain characterization, absorption/resorption currents measurements were carried out on cable sections that were cut from non-aged commercial cables provided by General Cable. The approximate length and capacitance of measured cables is $7 \mathrm{~cm}$ and $15 \mathrm{pF}$ respectively. Absorption and resorption currents were measured using a Keithley $6517 \mathrm{~A}$ and a polarization voltage of $1 \mathrm{kV}$ from its own stabilized DC source. No measurements were performed before minimization of noise under a level of approximately $\pm 20 \mathrm{fA}$. The determination of conductivity was performed by means of equation $5[3,4]$.

Frequency domain characterization has been performed by dynamic electrical analysis at the electric modulus and conductivity levels. In this case measurements were carried out on approximately $100 \mu \mathrm{m}$ thick sheets obtained from cable insulation by mechanical procedures.
Measurements were carried out at temperatures between 353 and $383 \mathrm{~K}$ on samples subjected to several annealing times in isothermal environment. Measurements of the electric modulus and conductivity were carried by means of a Novocontrol Dielectric Spectrometer and the real part of the conductivity was fitted to equation 9 usign $\sigma_{0}$, $n$ and $\omega_{p}$ as free parameters.

\section{RESULTS AND DISCUSSION}

\section{Time domain measurements}

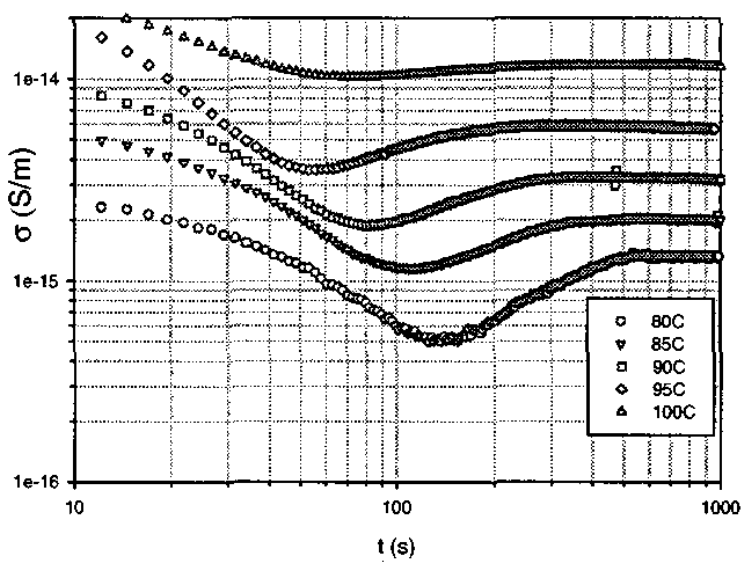

Figure 1: Cable insulation conductivity determined by absortion/resorption currents by means of equation 5

In figure 1 are plot the values of the conductivity resulting from Eq. 5 at several temperatures. It can be noticed that a stationary value is obtained for times as short as 5 minutes (or even before). An Arrhenius plot of stationary values of the conductivity obtained (Fig. 2) indicates a relation of the form

$$
\sigma=\sigma_{\tau 0} \mathrm{e}^{-\frac{E_{0}}{k T}}
$$

with $\sigma_{\tau 0}=12.95 \mathrm{~S} / \mathrm{m}$ and $E_{a}=0.53 \mathrm{eV}$

The absortion and resorption currents, $I_{a}$ and $I_{r}$ can be fitted to straight lines in a log-log scale, suggesting a dependence on time given by

$$
I(t)=I_{0} t^{-n_{c}}
$$

but in two separate intervales in each case. The first interval lasts up to $200 \mathrm{~s}$, and the second one corresponds to $t>200 \mathrm{~s}$ up to the end of the measurement. The parameters resulting from the fitting processes can be seen in tables 1 and 2 .

\section{Frequency domain measurements}

In Fig 4 we have plotted the values of the real part of the conductivity as a function of the frequency for several annealing times. It can be noticed that at frequencies below 


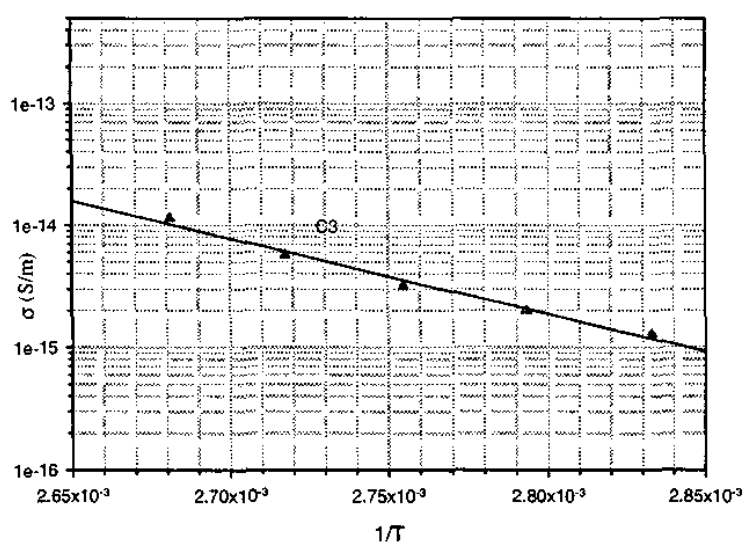

Figure 2: Arrhenius plot of conductivity

Table 1: Value of parameters obtained after fitting the measured current to equation 12. Two temporal intervals have been considered as indicated in the text

\begin{tabular}{|c|c|c|c|c|}
\hline Interval & \multicolumn{2}{|c|}{$I_{a}\left(80^{\circ} \mathrm{C}\right)$} & $I_{r}\left(80^{\circ} \mathrm{C}\right)$ \\
\hline & $I_{0}$ & $n_{c}$ & $I_{0}$ & $n_{c}$ \\
\hline$t<200 \mathrm{~s}$ & $2.13 \times 10^{-10}$ & 0.66 & $2.23 \times 10^{-10}$ & 0.69 \\
\hline$t>200 \mathrm{~s}$ & $6.25 \times 10^{-11}$ & 0.47 & $1.23 \times 10^{-10}$ & 1.44 \\
\hline
\end{tabular}

Table 2: Value of parameters obtained after fitting the measured current to equation 12 . Two temporal intervals have been considered as indicated in the text

\begin{tabular}{|c|c|c|c|c|}
\hline Interval & \multicolumn{2}{|c|}{$I_{a}\left(90^{\circ} \mathrm{C}\right)$} & \multicolumn{2}{c|}{$I_{r}\left(90^{\circ} \mathrm{C}\right)$} \\
\hline & $I_{0}$ & $n_{c}$ & $I_{0}$ & $n_{c}$ \\
\hline$t<200 \mathrm{~s}$ & $1.25 \times 10^{-10}$ & 0.45 & $7.08 \times 10^{-10}$ & 0.35 \\
\hline$t>200 \mathrm{~s}$ & $3.71 \times 10^{-11}$ & 0.30 & $2.57 \times 10^{-9}$ & 1.21 \\
\hline
\end{tabular}

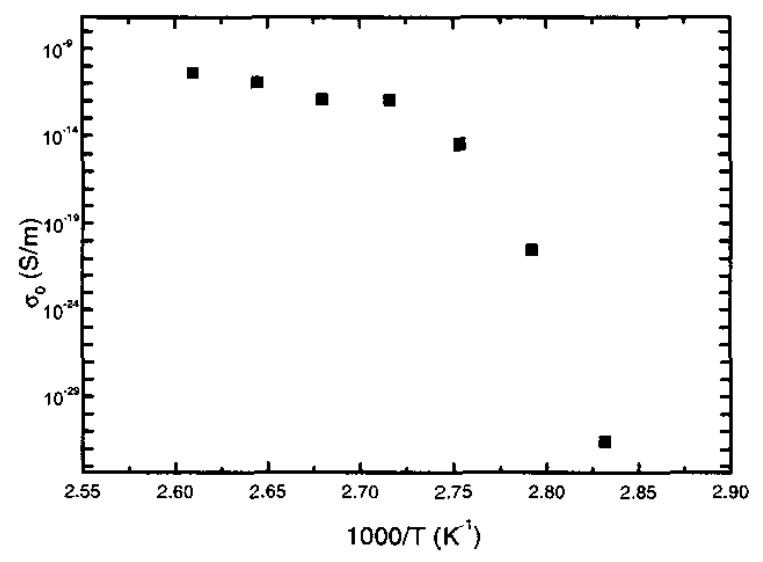

Figure 3: Arrhenius plot of the dc conductivity, $\sigma_{0}$, that results from fitting the real part of the conductivity to equation 9 approximately $1 \mathrm{~Hz}$ the real part of the conductivity at a fixed frequency increases with annealing time initialy, but eventually decreases. At higher frequencies a continuous decrease of $\sigma^{\prime}$ can be observed. At the electric modulus $\left(M^{*}=\left(\varepsilon^{*}\right)^{-1}\right)$ level, the behavior of the imaginary part of the electric modulus indicates the presence of a relaxation at frecuencies above $1 \mathrm{~Hz}$, that becomes more noticeable with annealing time (not shown).

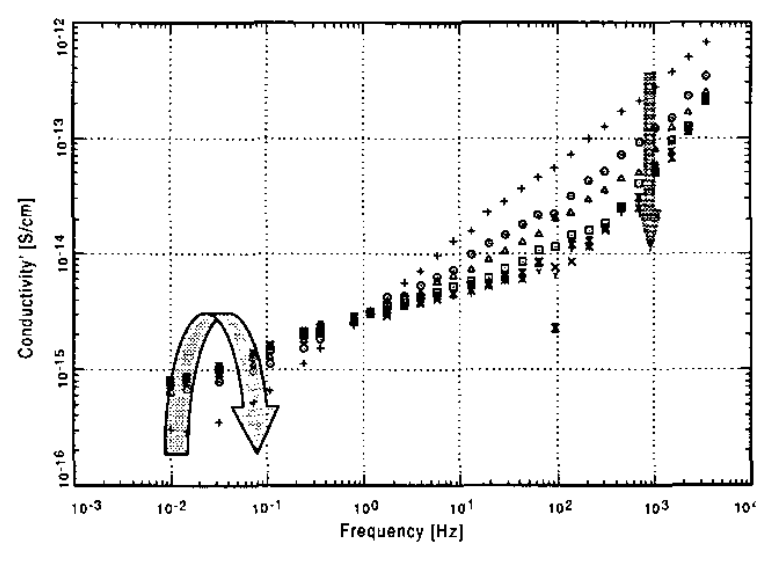

Figure 4: Real part of the conductivity at $373.15 \mathrm{~K}$ for several annealing times: $+0 \mathrm{~h} ; \circ 1 \mathrm{~h} ; \Delta 3 \mathrm{~h} ; \square 6 \mathrm{~h} ; \bowtie$ $12 \mathrm{~h} ; \times 18 \mathrm{~h} ; \Upsilon 36 \mathrm{~h}$

The values of $\sigma_{0}$ obtained grow with temperature (Fig. 3). A sharp increase can be observed at temperatures below $360 \mathrm{~K}\left(\approx 95^{\circ} \mathrm{C}\right)$ but for higher temperatures we can noticed that conductivity increases at a lower rate. It must be considered that DSC measurements indicates that this material melts over a wide temperature range, between 323 and $373 \mathrm{~K}$, and a pronounced peak can be noticed at approximately the temperature associated to the change in the temperature behavior. Therefore, the sharp increase of the conductivity can be related to the decrease of the crystalline fraction in the material, and once the melting process finishes there is a smooth increase of the conductivity with the temperature.

The behaviour of the crossover frequency $\omega_{p}$ with temperature (Fig. 5) is similar to the behaviour of $\sigma_{0}$. In the case of a ceramic material, experimental evidence of the equivalence of a characteristic time $\tau_{p}=1 / \omega_{p}$ and the Maxwell relaxation time

$$
\tau=\frac{\epsilon_{0} \epsilon}{\sigma}
$$

has been given[1]. In figure 5 it can be noticed that in our case, $\omega_{p}$ and $\sigma_{0}$ are proportional, suggesting a common physical mechanism, a lthough the ratio is not 1 .

The values obtained for the $n$ parameter range from 0.75 to 0.95 . This parameter characterizes the power-law conduction regime, which is associated with the slowing 


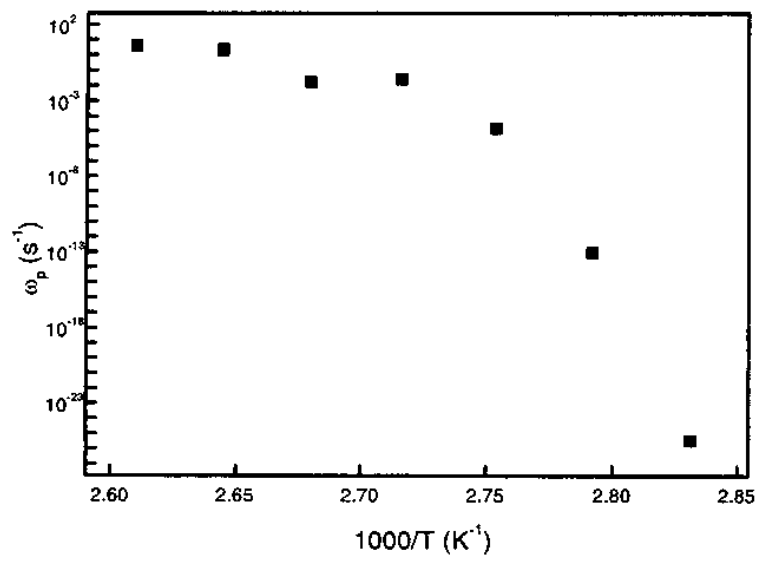

Figure 5: Arrhenius plot of the crossover frequency, $\omega_{p}$, that results from fitting the real part of the conductivity to equation 9

down of the relaxation process in the frequency domain as a result of cooperative effects, in the same way as the KWW function does in the time domain. An important connection between these two approaches stems from the coupling model of Ngai and Kannert[5]. This model predicts a power-law conductivity associated with the KWW relaxation function given by

$$
\sigma_{K W W}=B \exp \left(-E_{a} / k T\right) \omega^{1-\beta}
$$

Therefore, if any other contribution is sufficiently smaller than that of $\sigma_{\mathrm{KWW}} \mathrm{W}$, then the conductivity of the material may be described by $\sigma(\omega)_{K W W}[5]$.

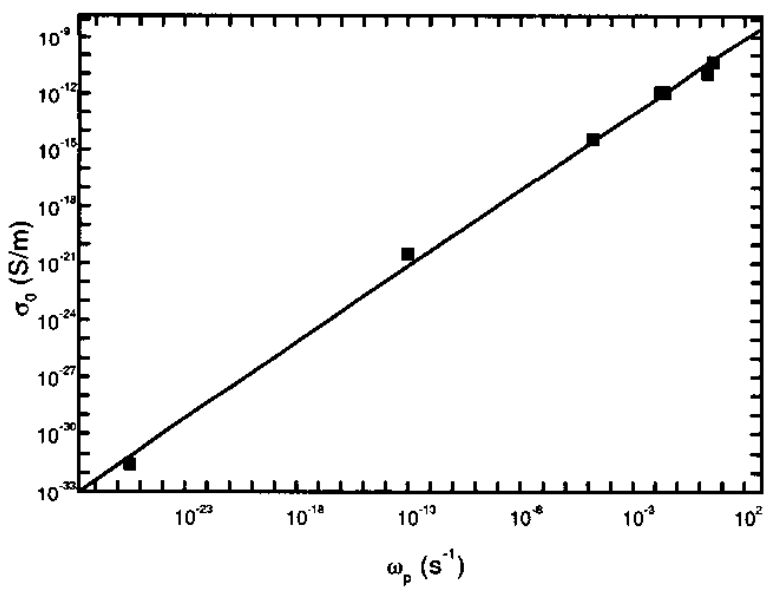

Figure 6: Conductivity versus crossover frequency

The sublinear dispersive ac conductivity observed may be associated with a KWW relaxation mechanism with $\beta=1-n$ where $n$ is the power-law exponent determined from $\sigma(\omega)$. The stretched exponential parameter $\beta$ may represent a correlation index of ionic motion, as the stretched exponential relaxation time has been associated with as slowing of the relaxation process that results from correlated ion hopping. For this reason, one would expect $\beta$ to be close to one for random Debye-like hops and close to zero for strongly correlated systems. Certain degree of correlation can, thus, be concluded from the observed values of $n$.

\section{CONCLUSIONS}

In the case of absortion and resortion currents measured in XLPE insulated mid-voltage cable sections two diferent time interval can be observed that can be fitted to a Curie-von Schweidler law.

DEA measurements show a dispersive conductivity that can be interpreted by means of a universal dynamic response. It has been determined that dc conductivity increases sharply over the temperature range along which the fusion takes place. At higher temperatures dc conductivity increases but at a lower rate. The values of the conductivity and the Maxwell relaxation time determined are proportional, so it may indicate a common physical mechanism. The value of the fractional exponent $n$ indicates that there is a certain degree of correlation in the ions hopping process.

\section{REFERENCES}

[1] C. León, M. L. Lucía, J. Santamaría," Correlated ion hopping in single-crystal yttria-stabilized zirconia",Phys. Rev. B, vol. 55 (2), pp. 882-887, 1997.

[2] D. P. Almond, A. R. West, R. J. Grant, "Temperature dependence of the a.c. conductivity of $\mathrm{Na} \beta$-alumina", Solid Stat. Comm., vol. 44, pp. 1277-1280, 1982.

[3] A.K. Jonscher, Dielectric relaxation in solids, London: Chelsea Dielectric Press, 1983.

[4] S. Hvidsten et al.,"Condition assessment of water treed service aged XLPE cables by dielectric response measurement", CIGRE'2000, paper 21-201.

[5] D. L. Sidebottom, P. F. Green, R. K. Brow, "Comparison of KWW and power law analyses of an ionconducting glass",J. Non-Cryst. Solids, vol. 183, pp.151-160, 1995.

Acknowledgments: Present work is financed by ID project Ref MAT 2001-2338-C02-01 (MCT Spain). The authors wish to thank General Cable for their contribution to the work (cable samples and useful suggestions). 